\title{
CHARACTERIZATION OF TIME-AVERAGED AND TEMPORAL TWO-PHASE FLOW STRUCTURES IN AERATED-LIQUID JETS USING X-RAY DIAGNOSTICS
}

\author{
KUO-CHENG LIN ${ }^{1}$, ALAN L. KASTENGREN ${ }^{2}$, SCOTT J. PELTIER $^{3} \&$ CAMPBELL D. CARTER $^{3}$ \\ ${ }^{1}$ Taitech, Inc., USA. \\ ${ }^{2}$ Argonne National Laboratory, USA. \\ ${ }^{3}$ Air Force Research Laboratory, USA.
}

\begin{abstract}
The structure of the two-phase flow inside the nozzle of an aerated-liquid injector and in the near field of the discharged plumes was experimentally explored with synchrotron x-ray diagnostics, including x-ray radiography, $\mathrm{x}$-ray fluorescence, and $\mathrm{x}$-ray high-speed imaging. One axisymmetric beryllium aeratedliquid injector featuring the inside-out aerating scheme was fabricated to mate with three aerating tube designs for the creation of two-phase flows. Water and nitrogen were doped with x-ray fluorescent elements at low concentrations to facilitate the x-ray diagnostics. Quantitative time-averaged liquid mass distributions for the two-phase mixture were successfully obtained from both radiography and fluorescence measurements. Averaged flow properties, such as liquid density and liquid velocity, at various cross-sections, were also derived from these measurements. Temporal formation and evolution of the two-phase mixture inside the aerated-liquid injector were also characterized with high-speed imaging. It was found that an annular flow is typically created in the two-phase mixture near the nozzle exit, despite the complex fluid dynamics in the liquid/gas interaction, flow passage volume change, and recirculation zone. The two-phase flow structures in the nozzle and the spray regions created from the present injector and aerating tube configurations are highly similar for a given injection condition. The major factor contributing to the similarity of the two-phase flow structures in the two regions may be the large area contraction ratio between the mixing chamber and the nozzle passage, which leads to a significant increase in flow speed and thus to aerodynamic stretching of the two-phase flow into fine structures.

Keywords: aerated-liquid jet, annular flow, beryllium, high-speed imaging, two-phase flow, x-ray radiography
\end{abstract}

\section{INTRODUCTION}

The study of two-phase flow is of great interest in applications in the propulsion, power, petroleum, civil, chemical, and nuclear industries, and effective qualitative and quantitative characterization of two-phase flow will further advance these applications. For instance, the aerated-liquid (or effervescent, or barbotage) injection scheme is a potential fuel delivery option for propulsion systems [1-3]. This injection scheme features the injection of a small amount of aerating gas into the liquid fuel to create a two-phase mixture inside the injector before discharge into a combustor. Improvement in liquid atomization, fuel/air mixing, and combustion can be achieved by creating a favourable two-phase mixture with an optimized injector design. In-situ diagnostics of the two-phase flow inside an axisymmetric metal aerated-liquid injector is therefore critical, not only to understand the complex two-phase flow structures, but also to provide measurements for nozzle design. Effective in-situ diagnostics tools are also needed in other applications involving two-phase flows.

Recently, several x-ray diagnostic techniques, including x-ray phase contrast imaging, $x$-ray radiography, and x-ray fluorescence, were utilized to characterize the near-field structures of aerated-liquid jets [4-7], in order to overcome the instrumentation challenges associated with interogating optically dense sprays with visible light sources. In particular, mass distributions of both liquid and gas phases within the discharged plumes were quantitatively characterized with 
great success using both x-ray radiography and x-ray fluorescence techniques. Liquid-based plume properties, such as density, velocity, and momentum flux averaged across plume crosssectional area, were readily derived from these measurements. The same experimental approach was later applied in the study of Peltier et al. [8] to explore the two-phase flow structures inside an aerated-liquid injector made out of beryllium. Beryllium has fairly low x-ray absorption and, therefore, allows for high transmittance of incident, transmitted, and emitted x-ray photons. In this study, the two-phase flow structures inside an aerated-liquid injector assembly featuring the inside-out aeration scheme and two aerating tube designs were explored.

While x-ray radiography and fluorescence diagnostics are capable of providing time-averaged flow properties, information on the temporal evolution of the two-phase flow is still needed, in order to address flow instability issues. The study of Lin et al. [9] recently added the X-ray highspeed imaging technique to the above-mentioned $\mathrm{x}$-ray diagnostics suite to investigate the temporal two-phase flow dynamics inside the nozzle passage region of an aerated-liquid injector featuring the outside-in aeration scheme. This injector design has several layers of metal in the liquid aeration region, however, so flow properties and dynamics in that region were not explored with $x$-ray diagnostics in that study.

The present study expands the work of Peltier et al. [8] by adding the x-ray high-speed imaging technique, in order to explore the time-averaged and temporal structures of the twophase flows inside an aerated-liquid injector. The injector assembly features the inside-out aerating scheme with variation in the aerating orifice diameter in the aerating tube. The region of interest reaches from the aerating area to the near field of the discharged plume.

\section{EXPERIMENTAL METHODS}

The experiment was conducted at the 7-BM beamline of the Advanced Photon Source (APS) at Argonne National Laboratory. The axisymmetric injector and liquid spray were positioned in the path of a small x-ray beam. Water and aerating gas were supplied into the aerated-liquid injector at the desired flow rates to form a two-phase mixture inside the injector before vertical discharge into a quiescent environment. The injector assembly was mounted on a traversing mechanism and translated in the $x-y$ plane. ( $x$ denotes the injector axial direction (positive down) and $y$ denotes the transverse direction.) A spray catchment assembly was placed below the injector to collect the liquid spray and to prevent the attachment of stray liquid droplets to the exterior of the injector body. The schematic in Fig. 1 illustrates the experimental setup.

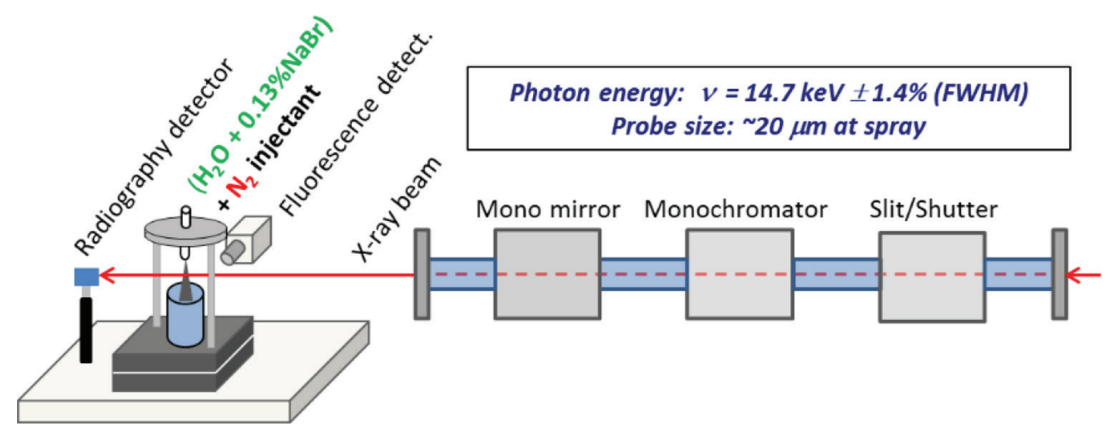

Figure 1: X-ray and injection stand setup at 7-BM beamline at Argonne National Laboratory. 


\subsection{Injector assembly}

The injector assembly features a so-called inside-out aerating configuration, with the aerating gas discharged through orifices in the aerating tube to create a two-phase mixture inside the injector. The injector was manufactured from beryllium ( $\mathrm{Be}>99.4 \%$ purity) due to its high x-ray transmittance. This injector was used in a previous study [8] and will be described here only briefly. The internal diameter of the injector, $d_{m}$, is $4.8 \mathrm{~mm}$. In the nozzle region, the inner passage has diameter, $d_{0}$, of $1.0 \mathrm{~mm}$ and length, $L$, of $10 \mathrm{~mm}$, leading to an $L / d_{0}$ of 10. The schematics in Fig. 2(a) illustrate the injector geometry (with a nominal aerating tube installed) and four flow regions of interest, namely aerating, mixing, nozzle, and spray regions. The aerating gas is added to the liquid within the aerating region. The mixing region between the end of the aerating tube and the nozzle entrance provides additional volume for the two-phase mixture to evolve. There is an area contraction ratio of 22:1 across the nozzle entrance.

Three aerating tube designs with different aerating orifice diameter, $d_{a}$, were utilized in the experiment. The critical dimensions of each tube, and the ratio between the total orifice area for aeration, $A_{a}$, and the internal cross-sectional area, $A_{t}$, for each tube, are listed in Table 1. The M2 aerating tube was used in Ref. [8]. These aerating tubes have the same placement pattern for the aerating orifices, as shown in Fig. 2(b). Each aerating tube is manufactured from stainless steel, with an outside diameter, $d_{t, o}$, of $3.2 \mathrm{~mm}$ and an internal diameter $d_{t, I}$, of $2.2 \mathrm{~mm}$. Each row of orifices is offset from the preceding row by $90^{\circ}$ and separated by a

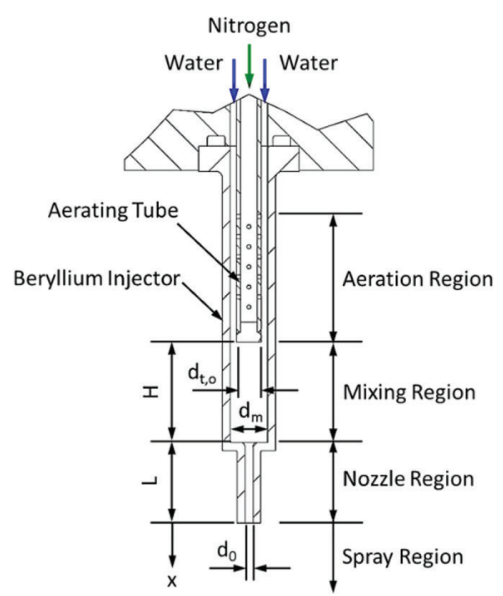

(a)

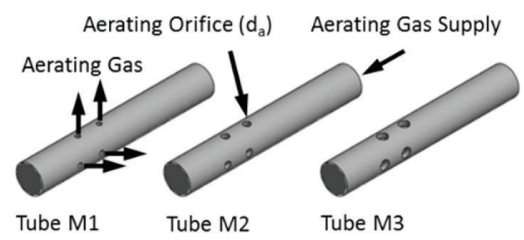

(b)

Figure 2: Schematics for (a) beryllium injector with aerating tube installed and (b) aerating tube geometry.

Table 1: Dimensions of aerating tubes.

\begin{tabular}{lllll}
\hline Tube & $\mathrm{d}_{\mathrm{a}}(\mathrm{mm})$ & Row & Orifice/Row & $\mathrm{A}_{\mathrm{a}} / \mathrm{A}_{\mathrm{t}}$ \\
\hline M1 & 0.51 & 4 & 2 & 0.44 \\
M2 & 0.76 & 4 & 2 & 1.00 \\
M3 & 1.02 & 3 & 3 & 1.77 \\
\hline
\end{tabular}


distance of $1.3 \mathrm{~mm}$. The distance between the tip of the aerating tube and the nozzle entrance was kept at $3.2 \mathrm{~mm}$. (The flow within the stainless steel aerating tube is inaccessible to x-ray diagnostics.) For the present study, the water mass flow rate, $m_{L}$, was kept at $18.2 \mathrm{~g} / \mathrm{s}$ and the nitrogen flow rate was also kept constant, to maintain a gas-to-liquid mass ratio, GLR, of $4 \%$.

\subsection{X-ray diagnostics}

The 7-BM beamline is configured for spray diagnostics using x-ray radiography, fluorescence, and tomography [10]. Nearly collimated, broadband x-ray emission is generated by a synchrotron bending magnet. The size of the x-ray beam is trimmed by a pair of slits, and the broadband emission is converted to a monochromatic beam by a double multilayer monochromator $(1.40 \% \Delta \mathrm{E} / \mathrm{E})$. Focusing is performed with a pair of $300 \mathrm{~mm}$ Kirkpatrick-Baez focusing mirrors, and the focus is placed $400 \mathrm{~mm}$ from the center of the horizontal focusing mirror. The $\mathrm{x}$-ray beam focused size is $5 \times 6 \mu \mathrm{m}$ FWHM $(\mathrm{V} \times \mathrm{H})$, but at the spray region the beam size is about $20 \mu \mathrm{m}$.

Time-averaged behavior of the two-phase flow was examined using x-ray radiography and fluorescence, using the procedures described in Ref. [8]. Pointwise x-ray radiography was performed by raster scanning across the injector geometry and spray region, with an averaging time of one second. X-ray transmission was measured using an unbiased silicon PIN diode. The attenuation of the $\mathrm{x}$-ray beam is described by the Beer-Lambert law:

$$
\frac{I}{I_{0}}=e^{-\alpha z}
$$

where $\mathrm{z}$ is the path length through the material, $\alpha$ is the linear attenuation coefficient, and $I$ and $I_{0}$ are the X-ray intensities measured before and after the sample, respectively.

$\mathrm{X}$-ray fluorescence from the liquid phase can be used to determine the distribution of liquid, by doping the water with bromine $(\mathrm{Br})$ atoms. Sodium bromide $(\mathrm{NaBr})$ was dissolved into the water at a concentration of $0.13 \%$ by mass. This concentration was monitored during the experiment by measuring the electrical conductivity of the water, which was approximately $1500 \mu \mathrm{S} / \mathrm{cm}$. The fluorescence signals were measured using a silicon drift diode detector, which measures $100 \mathrm{~mm}^{2}$ and was positioned approximately $100 \mathrm{~mm}$ from the injector and oriented $90^{\circ}$ to the $\mathrm{x}$-ray beam in order to minimize elastic $\mathrm{x}$-ray scattering. Oblique scattering of x-rays was mitigated by covering the detector with a Teflon shield.

The incident energy of the $\mathrm{x}$-ray beam was set to $14.7 \mathrm{keV}$ in order to surpass the absorption edges of $\mathrm{Br}(13.5 \mathrm{keV})$. When the incident photons impact the fluorescence tracers, photoionization of the core shell electrons occurs. An x-ray photon is emitted as the atom relaxes from this excited state, with the energy of the emitted photon being characteristic of the atomic number of the atom that emitted the photon. Thus, x-ray fluorescence is element-specific. The x-ray fluorescence photons are collected by the silicon drift diode, and the detector measures the number and energy of each $\mathrm{x}$-ray photon incident on the detector to form a spectrum of the fluorescence $\mathrm{x}$-rays. Further discussion of the x-ray fluorescence technique is given in Refs. [11] and [12].

In order to temporally resolve the two-phase flow structures, $\mathrm{x}$-ray high-speed images were also taken. The setup is similar to [9]. The raw x-ray emission from the bending magnet source (termed "white beam") was used to illuminate the sample with no filtering. The x-ray beam, measuring about $3 \mathrm{~mm}(\mathrm{H}) \times 8 \mathrm{~mm}(\mathrm{~W})$, was transmitted through the injector and then impinged on a YAG:Ce scintillator crystal $(500 \mu \mathrm{m}$ thick $)$ to convert the $\mathrm{x}$-ray image to 
visible light. A Photron SA-Z camera was operated at a framing rate of 271,554 fps (equalling the synchrotron ring frequency) and a shutter speed of $0.25 \mu \mathrm{s}$. At this framing rate, the maximum frame size for the camera is $144(\mathrm{H}) \times 256(\mathrm{~W})$ pixels. The field was then imaged 1:1, using a Nikon $105-\mathrm{mm} \mathrm{f} / 2.8$ macro lens, giving a spatial resolution of $20 \mu \mathrm{m} /$ pixel and a field of view of approximately $5.2 \mathrm{~mm}(\mathrm{H}) \times 2.9 \mathrm{~mm}(\mathrm{~V})$.

\subsection{Data reduction}

In this paper, the flow fields inside the beryllium nozzle are characterized by liquid density distribution. Details of the data reduction process can be found in Ref. [8] and will be briefly described here. The first parameter derived from the processed radiography or fluorescence signals is the equivalence path length $(E P L)$, which is the equivalent length for continuous distribution of the media of interest, such as liquid or gas phase, to generate identical radiography or fluorescence intensity measured from the two-phase mixture along the x-ray beam path. In the nozzle and spray regions, the water concentration is measured using the fluorescence signal from the $\mathrm{Br}$ tracer. Given the relatively high incident photon energy and the relatively short distance for the $\mathrm{x}$-ray to travel through the injector, the absorption of the incident beam would be reasonably small in both regions. For $\mathrm{Br}$ fluorescence photons at a high photon energy of $11.9 \mathrm{keV}$, the fluorescence signal is attenuated by only a few percent before reaching the detector. The procedure for determining the equivalent path length $(E P L)$ from the fluorescence measurement can be found in Ref. [8].

In the aerating and mixing regions inside the Be nozzle, the signal trapping was too large to use the above procedures. The liquid pathlengths, however, were large enough to give a reasonable radiography signal. Therefore, liquid $E P L$ was computed from the radiography data in both regions. The Beer-Lambert law in eqn (1) can be manipulated as follows:

$$
z=E P L=\frac{-\ln \left(I / I_{0}\right)}{\alpha}=\frac{-\ln \left(I / I_{0}\right)}{\beta \rho}
$$

where $\beta$ is the mass attenuation coefficient (in units of mass per unit area) and $\rho$ is the bulk material density. For liquid water with $0.13 \% \mathrm{NaBr}$ by mass $(0.10 \% \mathrm{Br}$ ions by mass) at 14.7 $\mathrm{keV}$, the mass attenuation coefficient is $\beta=0.1783 \mathrm{~m}^{2} / \mathrm{kg}$ [13]. The low concentration of $\mathrm{NaBr}$ has a negligible effect on the density, and therefore the value of $\rho$ for pure water is used here. The incident x-ray beam, however, is absorbed by both the two-phase mixture and the Be nozzle. In order to accurately compute the liquid EPL, the attenuation from the Be must first be resolved. Procedures to derive the actual attenuation by the two-phase mixture can be found in Ref. [8].

After obtaining the liquid EPL via fluorescence or radiography measurements, the line-ofsight averaged density $\rho(y)$ was computed with the following relationship:

$$
\rho(y)=\left(\frac{E P L}{D(y)}\right) \rho_{\text {water }}
$$

The chord length $D(y)$ through the two-phase mixture can be computed from the known geometry of the injector. In the spray region, the chord length $D(y)$ is a function of the plume cross-sectional width, where the plume is defined for regions with EPL greater than $1.0 \mu \mathrm{m}$.

For high-speed images, two approaches were utilized to enhance the image contrast ratio. First, the water solution was doped with $13 \%$ potassium iodide $(K I)$ by mass, in order to 
increase the absorption coefficient of the liquid phase under the white-beam x-ray. Second, each of the obtained images was normalized by an average reference image (a so-called flatfield image) at each imaging location and then saved as a 32-bit floating-point image using the ImageJ software package [14]. Image acquisition was carried out at several locations, in order to cover the entire x-ray accessible region within the Be nozzle. A total of 7,500 images were acquired at each location. Selected instantaneous and standard deviation images will be discussed here.

\section{RESULTS AND DISCUSSION}

\subsection{Time-averaged liquid phase distribution contours}

Contours of the line-of-sight density $(\rho(y))$ from eqn (3) are shown in Fig. 3 to illustrate the time-averaged two-phase flow structures inside the injector and within the near field of the discharged plume. At the $x=-24 \mathrm{~mm}$ location, which is upstream of the aerating orifices, the measured density is around $\rho(y)=1000 \mathrm{~kg} / \mathrm{m}^{3}$, indicating the absence of aerating gas, as expected. The density starts to decrease once flow enters the region with aerating orifice distribution, indicating the formation of the two-phase mixtures. For the test condition with the M1 aerating tube, the majority of the aerating gas stays close to the aerating tube near the end of the aerating region. For the other two injection conditions, however, the majority of the gas is distributed close to the injector wall, with a layer of liquid film flowing on the tube surface in the aerating region. This difference will be discussed with high-speed x-ray images later. Differences in flow structure in the mixing region can be observed among the three test conditions, mainly due to the presence of liquid film on aerating tube surface. High-density mixtures and even pure-liquid films are located close to the injector walls and the end of the mixing region for all three injection conditions. The two-phase mixture then expands in the 22:1 area reduction from the mixing region to the nozzle region, to reach a low density as it enters the nozzle region. The flow structures are highly similar in the nozzle region. The

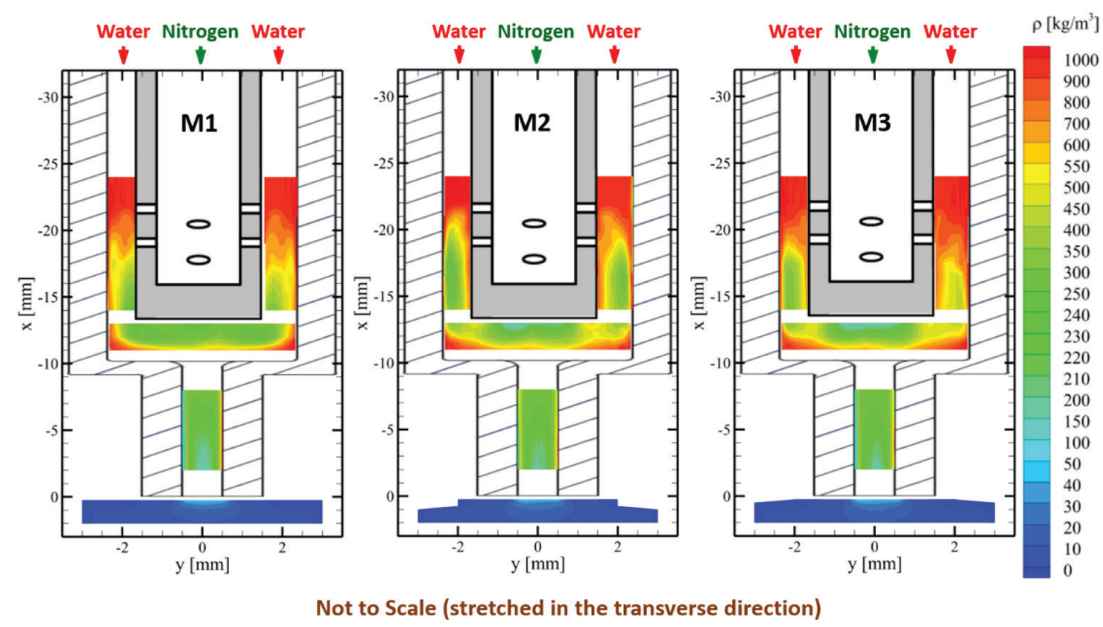

Figure 3: Contours of line-of-sight averaged density $\rho(y)$ for injection conditions with various aerating tubes. $m_{L}=18.2 \mathrm{~g} / \mathrm{s}, G L R=4 \%$. 
two-phase mixture eventually exits the nozzle as a highly dispersed plume with mixture density less than $100 \mathrm{~kg} / \mathrm{m} 3$. As can be seen in Fig. 3, the present X-ray diagnostics are capable of providing important insights into the characteristics and behaviour of the two-phase flow inside an axisymmetric injector. In particular, time-averaged liquid mass distributions inside the axisymmetric injector can be clearly depicted.

The evolution of the two-phase mixture is further illustrated in Fig. 4 by the line-of-sight liquid density profiles at various axial locations for the M1 aerating tube. Please note that only the left half of the aerating region is depicted in Fig. 4(a). A two-phase flow with reduced mixture density is quickly created by the aerating gas delivered through the aerating orifices. Instead of creating a uniform two-phase mixture near the end of the aerating region, however, the majority of the liquid is squeezed to the Be nozzle wall by the compressible aerating gas. The aerating gas is mainly distributed close to the aerating tube, creating a gas-dominant mixture with reduced mixture density. Near the entrance of the mixing region at $x=$ $-13.0 \mathrm{~mm}$ in Fig. 4(b), the gas-dominant mixtures from the annular aerating region quickly merge, creating a large low-density region within the wake of the aerating tube. This low-density region persists until the flow enters the nozzle region. The liquid-dominant mixtures stay close to the Be nozzle wall from $x=-13$ to $-11.5 \mathrm{~mm}$, and eventually accumulate near the bottom of the mixing chamber, as indicated by the elevated line-of-sight density at $x=-11 \mathrm{~mm}$. Down to this axial location, the two-phase mixture is still fairly non-uniform.

Moving down from the nozzle entrance, the liquid is distributed further away from the core flow, with the centerline mixture density decreasing from approximately 280 to $180 \mathrm{~kg} / \mathrm{m}^{3}$ between $x=-8.0$ and $-2.0 \mathrm{~mm}$ in Fig. 4(c). It appears that the structure of the two-phase

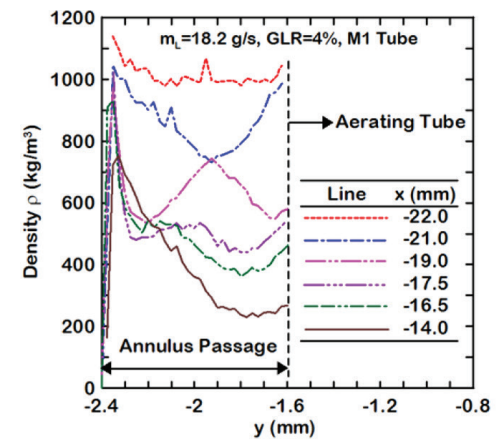

(a)

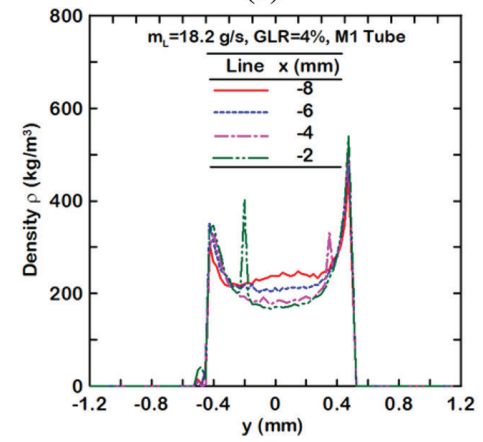

(c)

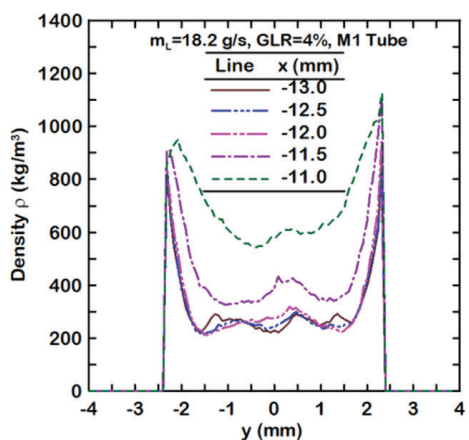

(b)

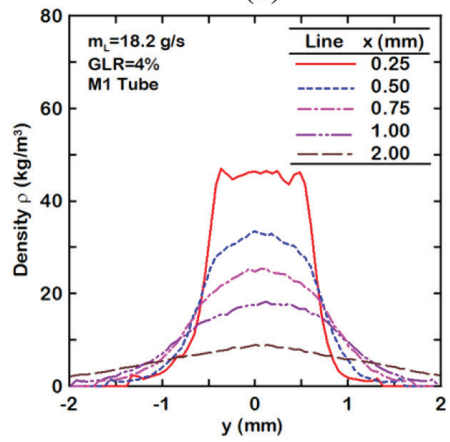

(d)

Figure 4: Line-of-sight density profiles in (a) aerating region (showing only left half), (b) mixing region, (c) nozzle region, and (d) spray region. 
mixture is more or less annular, with a liquid-dominant mixture along the nozzle wall and gas-dominant mixture in the core flow. The line-of-sight liquid density profiles in Fig. 4(c) can be further processed with the Abel inversion scheme to obtain spatially-resolved liquid distributions, under the assumption of fully axisymmetric flow in the future. X-ray interference from stray droplets attached to the nozzle exterior wall can be seen in the unexpected spike in liquid density at the $x=-2 \mathrm{~mm}$ location. After the nozzle exit, the line-of-sight density of the two-phase mixture decreases to around $50 \mathrm{~kg} / \mathrm{m}^{3}$, with the annular-like structure quickly decaying at the $x=0.25 \mathrm{~mm}$ location in Fig. 4(d). At the further downstream locations, the spray plume exhibits Gaussian-like density profiles with reduced peak values. Please note that the calculation of the mixture density in the spray region is based on an arbitrarily-selected spray boundary for the chord length $(D(y))$ determination in eqn (3). The calculated density in the spray region, therefore, is not definitive.

\subsection{Effects of orifice size on aerating tube}

The effects of aerating orifice diameter on the two-phase flow structures are further illustrated by the line-of-sight liquid density profiles at selected axial locations for each region in Fig. 5. For the present aerating tube designs, differences in two-phase flow structures can be observed in the aerating region and the upstream portion of the mixing region, i.e. $x<-11 \mathrm{~mm}$. Once the two-phase flow enters the nozzle, the time-averaged line-of-sight liquid density profiles exhibit highly similar flow structures in both nozzle and spray regions for the present injection conditions. The similarity between the two-phase flow structures in both regions may result primarily from the 22:1 area contraction ratio between the mixing chamber and the nozzle passage, as will be further discussed later. The macroscopic features of the spray plume, such as the plume width, are also highly similar.

\subsection{Temporal two-phase flow characterization with high-speed imaging}

Figure 6 shows composite images from uncorrelated individual instantaneous images and standard deviation images taken at various locations to illustrate the two-phase flow structures inside the beryllium nozzles with various aerating tubes at the same injection condition. Please note that the present camera setup has a spatial resolution of $20 \mu \mathrm{m}$ per pixel. Any interface features below this spatial resolution cannot be resolved. Nonetheless, the images in Fig. 6 show that large-scale structures with identifiable interfaces are mainly located in the aerating and mixing regions. The aerating gas is discharged into the liquid annulus flow in the form of gas streams, not individual tiny gas bubbles, in the present aerating tube designs. The trajectory of the furthest upstream gas stream correlates with the aerating orifice diameter for a given gas flow rate. The small aerating orifice on the M1 tube generates a high-speed (or highmomentum) gas stream, which penetrates deeper into the liquid crossflow at a given liquid flow rate. This feature is also clearly depicted in the standard deviation images. The accumulated liquid in the corners of the mixing region and the slight misalignment of the aerating tube can also be clearly observed in these x-ray images. The length scale of the two-phase structures dramatically decreases in the nozzle passage section, probably due to the 22:1 area contraction ratio between the mixing chamber and the nozzle passage. This relatively large area contraction forces the two-phase mixture to quickly accelerate, and stretches the twophase flow structures in the mixing region into small-scale structures. Consequently, no identifiable interface can be effectively discriminated by the present high-speed camera setup 

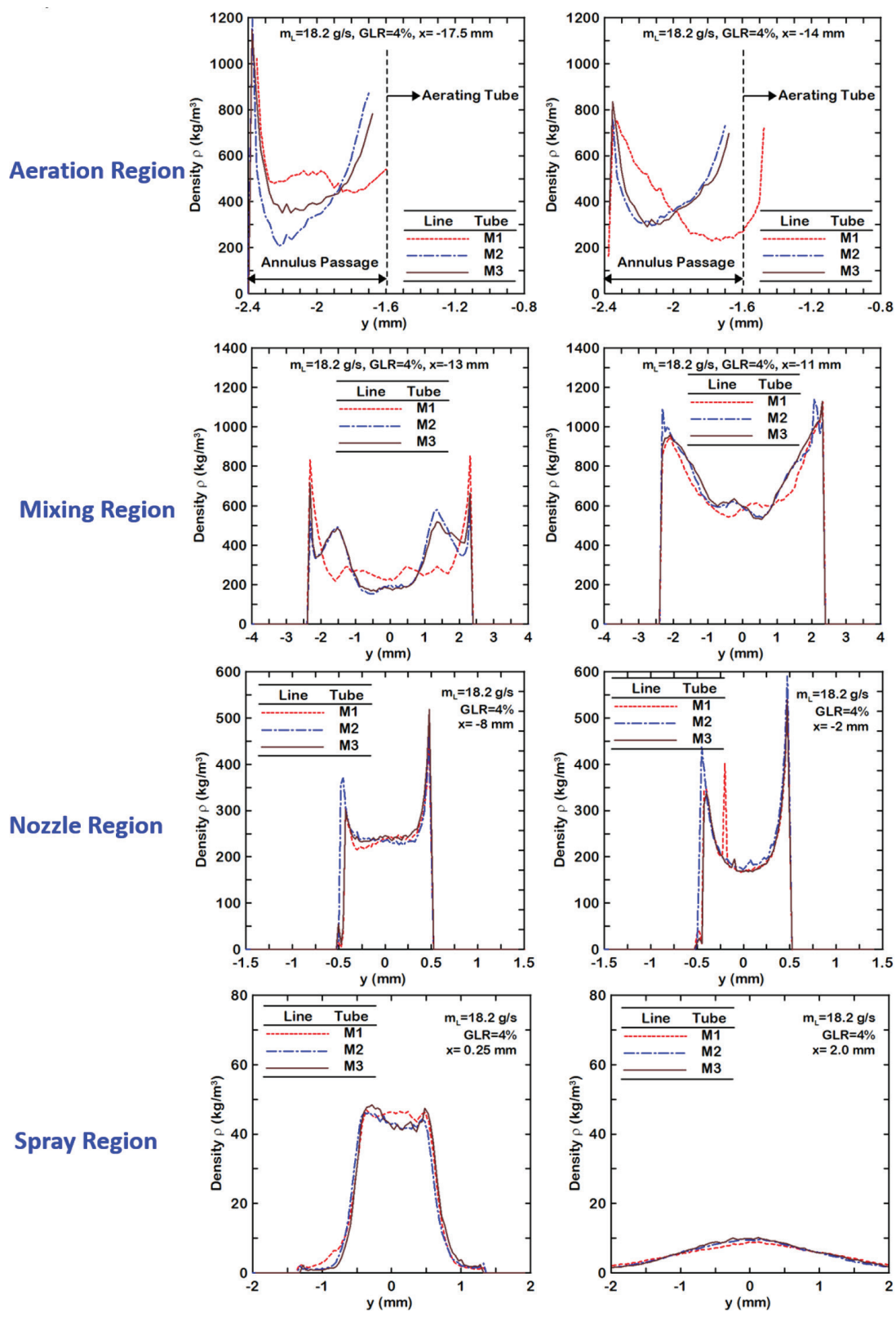

Figure 5: Selected line-of-sight liquid density profiles with various aerating tubes.

without further improvement in spatial resolution, camera shutter speed, and interface contrast enhancement. Nonetheless, the obtained images can be compiled to create movies at a low playback speed to further understanding of the temporal evolution of the two-phase flows within the nozzles. 


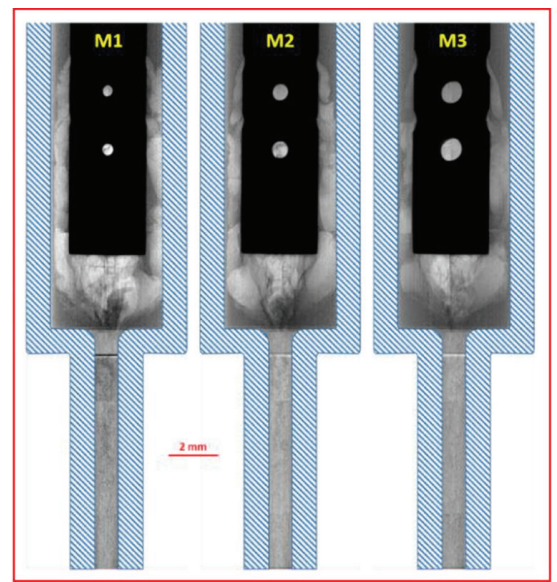

(a)

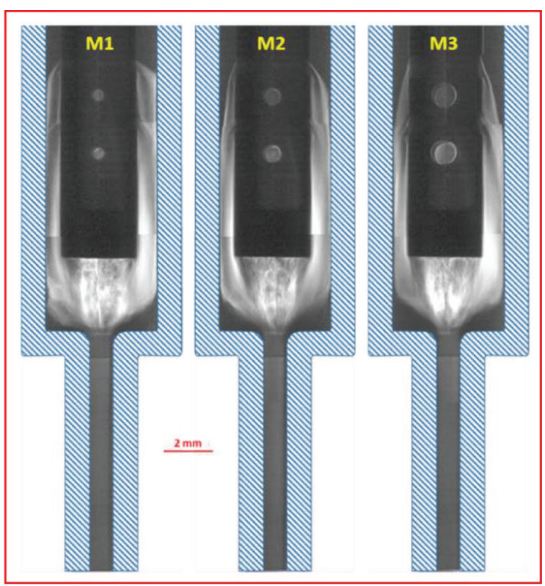

(b)

Figure 6: Composites of (a) instantaneous images and (b) standard deviation images from high-speed camera.

\subsection{Averaged flow properties}

The averaged mixture density at each axial $(x)$ location can be calculated by directly integrating the line-of-sight averaged density in the y-direction in the mixing, nozzle, and spray regions. In the aerating region, instead, the averaged density is calculated by transforming the line-of-sight averaged density $\rho(y)$ into a radial distribution $\rho(r)$, and then integrating azimuthally within the annulus flow passage. Given the density distributions described above, the weighted liquid velocity $u(x)$ can then be calculated as follows:

$$
u(x)=\frac{m_{L}}{\sum[\rho(y) D(y) \Delta y]}=\frac{m_{L}}{T I M}
$$

where TIM is the time-averaged integrated mass of the liquid computed at each axial position.

Figure 7 shows the evolution of the averaged density $(\rho)$, velocity $(u)$, and momentum flux $\left(\rho u^{2}\right)$ along the injector axis. The averaged density decreases continuously in the aerating region, due to gas addition into the annulus. Density of the two-phase mixture then exhibits a sudden decrease across the entrance of the mixing region, due to an abrupt flow passage area increase and the existence of gas-dominant mixtures in the wake of the aerating tube. Subsequently, the averaged density gradually increases toward the nozzle region, due to liquid accumulation on the wall and in the corners of the mixing chamber. The averaged density exhibits a significant decrease across the entrance of the nozzle region due to the 22:1 area contraction ratio. A significant increase in averaged liquid velocity is observed across the nozzle entrance, resulting in a high rate of aerodynamic stretch, which breaks up the twophase flow into small-scale structures. As the two-phase mixture flows toward the nozzle exit orifice, the averaged density (velocity) gradually decreases (increases). The averaged velocity further increases upon discharge, due to the expansion of the choked two-phase flow. The rapid expansion of the two-phase mixture in the ambient (spray region) creates a huge decrease in density, leading to a decrease in the averaged momentum flux in the spray region. 


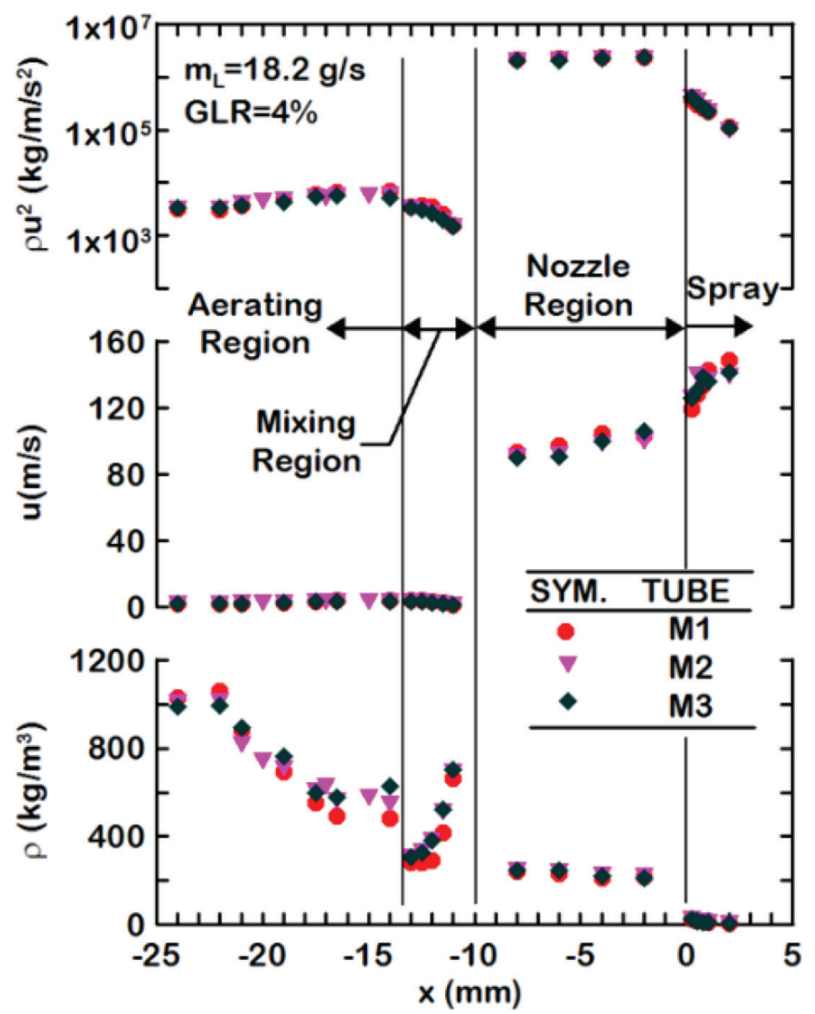

Figure 7: Axial distributions of averaged liquid density $(\rho)$, velocity $(u)$, and momentum flux $\left(\rho u^{2}\right)$ for the injection condition with $m_{L}=18.2 \mathrm{~g} / \mathrm{s}$ and $G L R=4 \%$.

The maximum momentum flux of the two-phase mixture appears near the nozzle exit. No significant differences in the averaged flow properties can be observed in the mixing, nozzle, and spray regions for the present aerating tube designs.

\section{CONCLUSIONS}

Structures of the two-phase mixture inside an axisymmetric aerated-liquid injector and within the near field of the discharged plumes were explored, using x-ray radiography, $\mathrm{x}$-ray fluorescence, and x-ray high-speed imaging, at the Advanced Photon Source at Argonne National Laboratory. Beryllium was selected as the nozzle material, due to its high X-ray transmittance. The aerated-liquid injector features an inside-out aerating scheme with an aerating tube submerged in the liquid stream for aerating gas delivery. The test matrix consists of three aerating tube designs at the same injectant flow rates. Quantitative time-averaged liquid mass distributions for the two-phase mixture were successfully obtained from both radiography and fluorescence measurements. Averaged flow properties, such as liquid density and liquid velocity at various cross-sections, were also derived from these measurements. Temporal formation and evolution of the two-phase mixture inside the aerated-liquid injector were also characterized with high-speed imaging. It was found that an annular flow is typically created in the two-phase mixture near the nozzle exit, despite the complex fluid dynamics in the liquid/gas interaction, flow passage volume change, and recirculation zone. The two-phase flow structures in the nozzle and the spray regions created from the present injector and aerating 
tube configurations are highly similar for a given injection condition. The major factor contributing to the similarity of the two-phase flow structures in the two regions may be the large area contraction ratio between the mixing chamber and the nozzle passage, which leads to a significant increase in flow speed and thus to aerodynamic stretching of the two-phase flow into fine structures.

\section{ACKNOWLEDGEMENTS}

This work was sponsored by the AFRL/Aerospace Systems Directorate under contract number FA8650-14-D-2316 and by the Air Force Office of Scientific Research (AFOSR). Use of the Advanced Photon Source at Argonne National Laboratory was supported by the U. S. Department of Energy, Office of Science, Office of Basic Energy Sciences, under Contract No. DE-AC02-06CH11357.

\section{REFERENCES}

[1] Lefebvre, A.H., Wang, X.F. \& Martin, C.A., Spray characteristics of aerated-liquid pressure atomizers. Journal of Propulsion and Power, 4, pp. 293-298, 1988. https://doi.org/10.2514/3.23066

[2] Sovani, S.D., Sojka, P.E. \& Lefebvre, A.H., Effervescent atomization. Progress in Energy and Combustion Science, 27, pp. 483-521, 2001.

https://doi.org/10.1016/s0360-1285(00)00029-0

[3] Mathur et al., Liquid JP-7 combustion in a scramjet combustor. AIAA Paper 2000-3581, August, 2000.

[4] Lin, K.-C., Rajnicek, C., McCall, J., Carter, C. \& Fezzaa, K., Investigation of pure- and aerated-liquid jets using $\mathrm{X}$-ray phase contrast imaging technique. Nuclear Instruments and Methods in Physics Research Section A, 649(1), pp. 194-196, 2011. https://doi.org/10.1016/j.nima.2010.11.122

[5] Lin, K.-C., Carter, C., Smith, S. \& Kastengren, A., Exploration of aerated-liquid jets using x-ray radiography. AIAA Paper 2012-0347, January, 2012.

[6] Lin, K.-C., Carter, C., Smith, S. \& Kastengren, A., Exploration of near-field plume properties for aerated-liquid jets using x-ray radiography. AIAA Paper 2014-1183, January, 2014.

[7] Lin, K.-C., Carter, C., Smith, S. \& Kastengren, A., Characterization of aeratedliquid jets using simultaneous $\mathrm{x}$-ray radiography and $\mathrm{x}$-ray fluorescence measurements. Atomization and Sprays, to be published.

[8] Peltier, S., Lin, K.-C., Carter, C. \& Kastengren, A., Characterization of flow structures inside an aerated-liquid injector using X-ray diagnostics. Experiments in Fluids, 58, p. 111, 2017. https://doi.org/10.1007/s00348-017-2380-4

[9] Lin, K.-C., Kastengren, A.L., Carter, C. \& Donbar, J., Exploration of two-phase flow structures in aerated-liquid jets using beryllium nozzles and $\mathrm{x}$-ray fluorescence techniques. AIAA Paper 2017-0825, January, 2017

[10] Kastengren, A.L., Powell, C.F., Arms, D., Dufresne, E. \& Wang, J., Spray diagnostics at the advanced photon source 7-BM beamline," Proceedings of the 22nd Annual Conference on Liquid Atomization and Spray Systems, May, 2010.

[11] Kastengren, A.L. \& Powell, C.F., Synchrotron x-ray techniques for fluid dynamics. Experiments in Fluids, 55(3), Article 1686, 2014.

https://doi.org/10.1007/s00348-014-1686-8 
[12] Kastengren, A.L., X-ray fluorescence as a gas-phase mixing diagnostic. Proceeding of Central States Section of the Combustion Institute Spring Technical Meeting, March 2014.

[13] XCOM: Photon Cross Sections Database, NIST Standard Reference Database 8 (XGAM), available at: http://www.nist.gov/pml/data/xcom/index.cfm

[14] Schneider, C.A., Rashband, W.S. \& Eliceiri, K.W., NIH image to ImageJ: 25 years of image analysis. Nature Methods, 9, pp. 671-675, 2012.

https://doi.org/10.1038/nmeth.2089 